\title{
Papillary thyroid carcinoma associated with silent paraganglioma: An atypical MEN Syndrome?
}

\author{
Ozlem TARCIN ${ }^{1}$, Dilek YAZICI ${ }^{2}$, Asli UNAL ${ }^{1}$, Nilgun DEMIRAG ${ }^{1}$ \\ ${ }^{1}$ Baskent University Hospital, Endocrinology and Metabolism, Istanbul, TURKEY \\ ${ }^{2}$ Marmara University Hospital, Endocrinology and Metabolism, Istanbul, TURKEY
}

\begin{abstract}
Case: A 51 year-old-woman was referred to endocrinology outpatient clinic due to a thyroid nodule $9 \mathrm{~mm}$ in diameter. Fine needle aspiration (FNA) biopsy revealed papillary thyroid carcinoma and she was directed to surgery. The surgeon decided to perform subtotal thyroidectomy due to the small nodule size. Pathologic examination showed the histology to be consistent with tall cell carcinoma. Complementary total thyroidectomy was proposed to the patient but she did not accept the second operation. During close follow-up for 2 years, thyroglobulin levels were high about $4 \mathrm{mg} / \mathrm{dl}$ and there were reactive cervical lymphadenopathies which were $2.5 \mathrm{~cm}$ in diameter. PET/CT was performed to be sure about any metastasis. Surprisingly, a $7 \times 5 \mathrm{~cm}$ hypodense lesion, with a high FDG uptake was detected in the mid-upper abdominal region and the mass was suspected to be lymphoma first (Figure 1). In the abdominal CT scan, $6.7 \times 5.9 \times 6.6 \mathrm{~cm}$ retroperitoneal mass was located at the aortacaval region (Figure 2). The patient was consultated with the hematology department and they recommended FNA biopsy to be performed from the mass. During the biopsy procedure, no clinical symptoms were evident and the pathologic diagnosis was reported to be a paraganglioma.24-hours urine fractioned

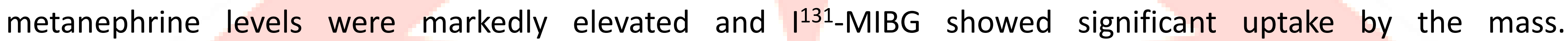
Chromogranin-A level was very high whereas PTH and calcitonin were in normal ranges. Surgery was performed for the abdominal paraganglioma after the patient was prepared for the operation with alpha-blocker agents although she did not have a hypertensive attack or any clinical finding before. The excised mass was $10 \mathrm{~cm}$ in diameter and the pathology was reported to be grade I neuroendocrine tumor without Ki67 proliferation.

Three months after the operation, 24-hours urine fractioned metanephrines and MIBG scan were normal.

Consciously, thyroid protection with iodine was not carried out during MIBG procedures so thyroid gland shrank in size. Radioactive iodine ablation therapy was performed for thyroid papillary carcinoma successfully. Thyroglobulin level decreased after ablation.

The patient remembered that her mother had a mass in her neck which could have been paraganglioma. SDHD/SDHB and RET mutation analyses were planned and we are still waiting for the results.

\section{Discussion:}

Papillary thyroid carcinoma associated with pheochromocytoma had been reported previously both sporadically and with MEN syndromes but rarely with a paraganglioma. SDHD or SDHB mutation (for paraganglioma) and RET proto-oncogene (for MEN) analysis are important for diagnosis of genetic basis of the diseases. The relationship between these two lesions could not still be determined in our case. Recently, a case with papillary thyroid carcinoma associated with paraganglioma and Dandy-Walker Malformation was reported whose SDHD mutation positive ${ }^{1}$. The authors have noted that SDHx mutations can cause familial paraganglioma syndromes. In our case if mutation analysis reveals positive results, these combinations could be explained as an atypical MEN syndrome or a subtype of MEN syndrome.
\end{abstract}

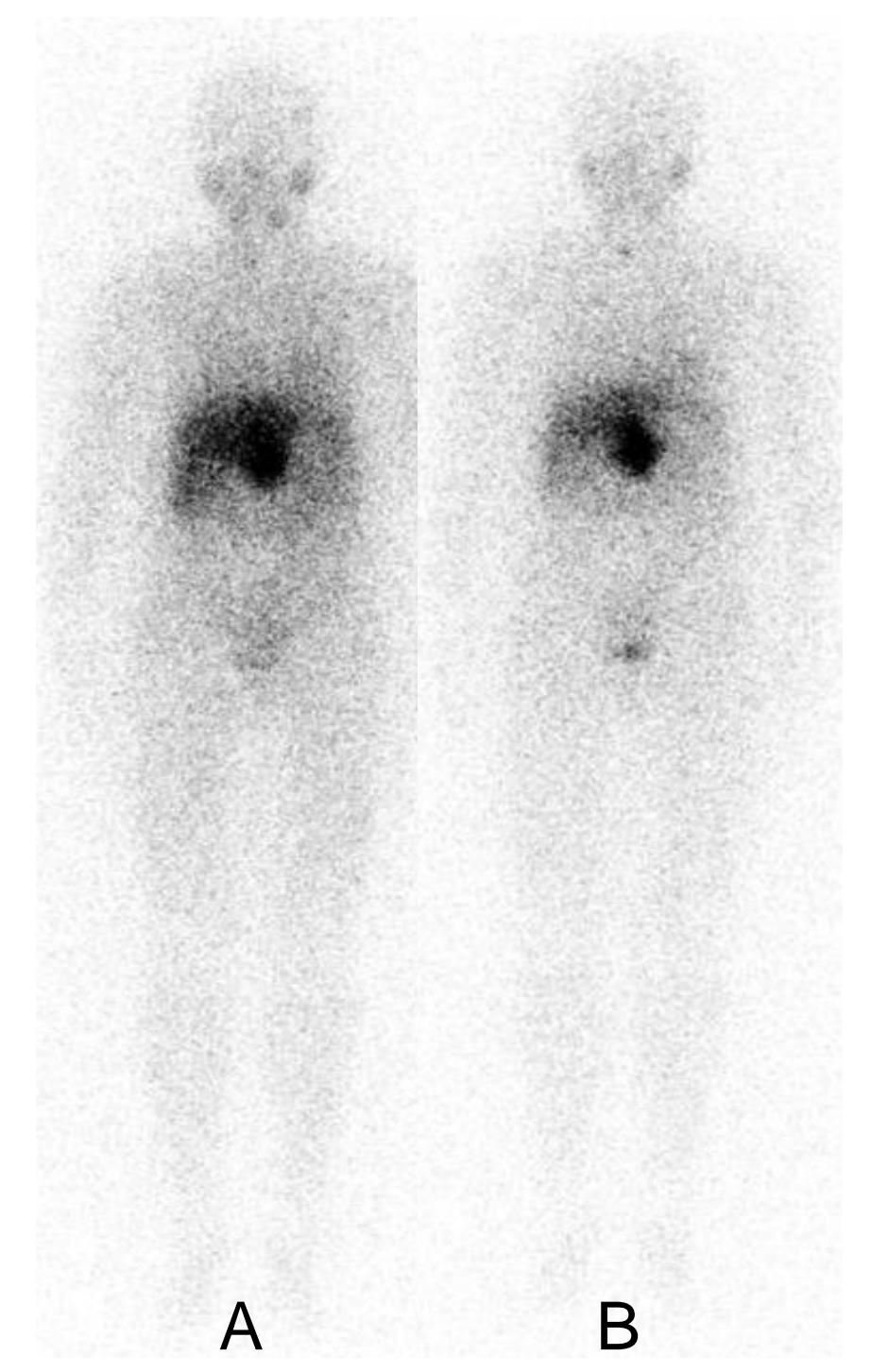

Figure 1: High FDG uptake of mass in PET/CT

(A: anterior image, $B$ : posterior image)

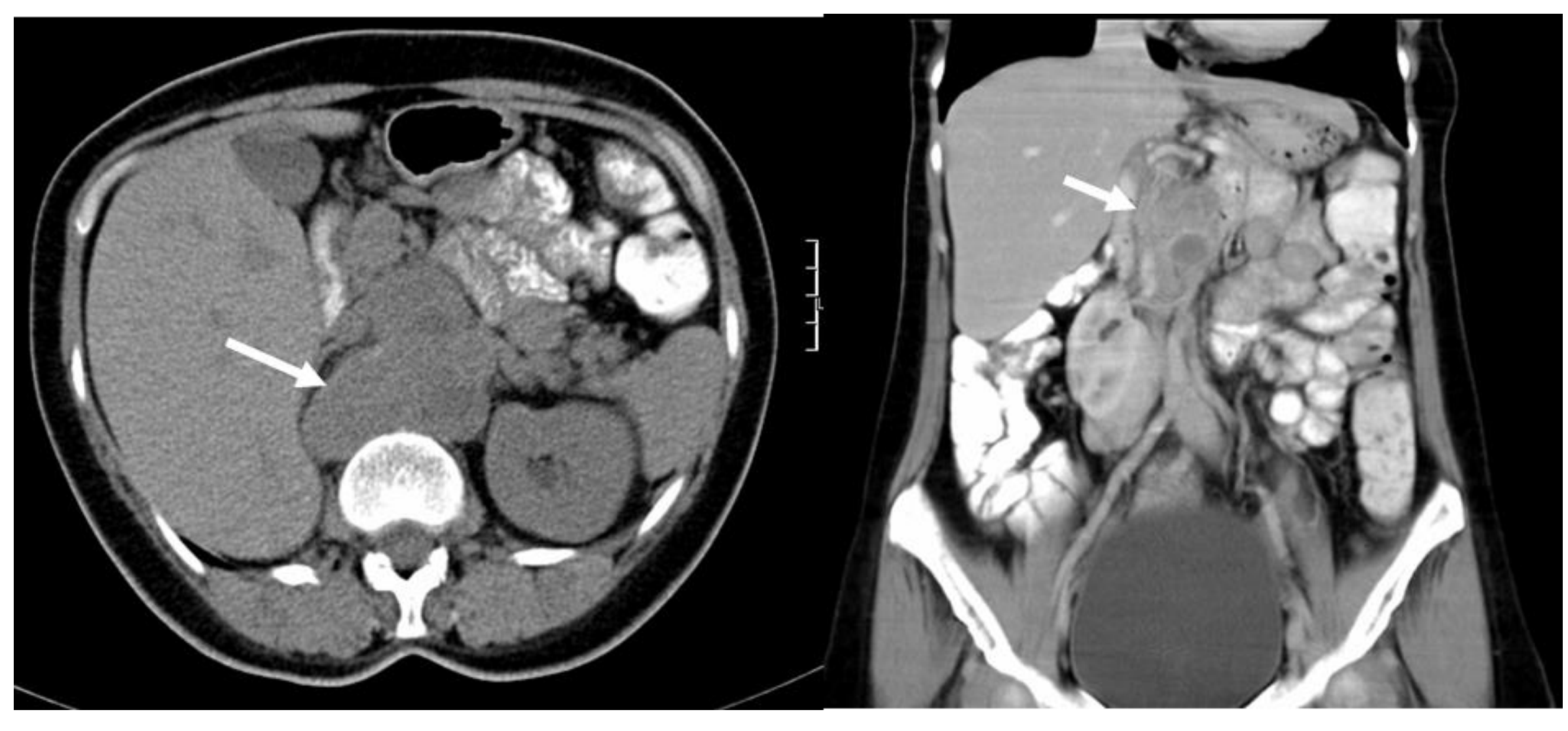

Figure 2: The arrows show retroperitoneal aortacaval mass in abdominal CT (transverse and coronal images) 